\title{
Analysis of the Spatial Distribution of Galaxies by Multiscale Methods
}

\author{
J-L. Starck \\ DAPNIA/SEDI-SAP, Service d'Astrophysique, CEA-Saclay, 91191 Gif-sur-Yvette, France \\ Email: jstarck@cea.fr
}

\section{J. Martínez}

Observatori Astronòmic de la Universitat de València, Edifici d'Instituts de Paterna, Apartat de Correus 22085, 46071 València, Spain

Email:vicent.martinez@uv.es

\section{L. Donoho}

Department of Statistics, Stanford University, Sequoia Hall, Stanford, CA 94305, USA Email:donoho@stanford.edu

\section{O. Levi}

Department of Statistics, Stanford University, Sequoia Hall, Stanford, CA 94305, USA

Email:levio@bgumail.bgu.ac.il

\section{P. Querre}

DAPNIA/SEDI-SAP, Service d'Astrophysique, CEA-Saclay, 91191 Gif-sur-Yvette, France Email:philippe.querre@irsn.fr

\section{E. Saar}

Department of Cosmology, Tartu Observatory, Toravere 61602, Estonia

Email:saar@aai.ee

Received 17 June 2004; Revised 17 February 2005

Galaxies are arranged in interconnected walls and filaments forming a cosmic web encompassing huge, nearly empty, regions between the structures. Many statistical methods have been proposed in the past in order to describe the galaxy distribution and discriminate the different cosmological models. We present in this paper multiscale geometric transforms sensitive to clusters, sheets, and walls: the 3D isotropic undecimated wavelet transform, the 3D ridgelet transform, and the 3D beamlet transform. We show that statistical properties of transform coefficients measure in a coherent and statistically reliable way, the degree of clustering, filamentarity, sheetedness, and voidedness of a data set.

Keywords and phrases: galaxy distribution, large-scale structures, wavelet, ridgelet, beamlet, multiscale methods.

\section{INTRODUCTION}

Galaxies are not uniformly distributed throughout the universe. Voids, filaments, clusters, and walls of galaxies can be observed, and their distribution constrains our cosmological theories. Therefore we need reliable statistical methods to compare the observed galaxy distribution with theoretical models and cosmological simulations.

The standard approach for testing models is to define a point process which can be characterized by statistical descriptors. This could be the distribution of galaxies of a specific type in deep redshift surveys of galaxies (or of clusters of galaxies). ${ }^{1}$ In order to compare models of structure formation, the different distribution of dark matter particles

\footnotetext{
${ }^{1}$ Making 3D maps of galaxies requires knowing how far away each galaxy is from Earth. One way to get this distance is to use Hubble's law for the expansion of the universe and to measure the shift, called redshift, to redder colors of spectral features in the galaxy spectrum. The greater the redshift, the larger the velocity, and, by Hubble's law, the larger the distance.
} 
in N-body simulations could be analyzed as well, with the same statistics.

The two-point correlation function $\xi(r)$ has been the primary tool for quantifying large-scale cosmic structure [1]. Assuming that the galaxy distribution in the Universe is a realization of a stationary and isotropic random process, the two-point correlation function can be defined from the probability $\delta P$ of finding an object within a volume element $\delta V$ at distance $r$ from a randomly chosen object or position inside the volume: $\delta P=n(1+\xi(r)) \delta V$, where $n$ is the mean density of objects. The function $\xi(r)$ measures the clustering properties of objects in a given volume. It is zero for a uniform random distribution, positive (resp., negative) for a more (resp., less) clustered distribution. For a hierarchical clustering or fractal process, $1+\xi(r)$ follows a power-law behavior with exponent $D_{2}-3$. Since $\xi(r) \sim r^{-\gamma}$ for the observed galaxy distribution, the correlation dimension for the range where $\xi(r) \gg 1$ is $D_{2} \simeq 3-\gamma$. The Fourier transform of the correlation function is the power spectrum. The direct measurement of the power spectrum from redshift surveys is of major interest because model predictions are made in terms of the power spectral density. It seems clear that the real space power spectrum departs from a single power-law ruling out simple unbounded fractal models [2]. The two-point correlation function can been generalized to the $\mathrm{N}$-point correlation function $[3,4]$, and all the hierarchy can be related with the physics responsible for the clustering of matter. Nevertheless they are difficult to measure, and therefore other related statistical measures have been introduced as a complement in the statistical description of the spatial distribution of galaxies [5], such as the void probability function [6], the multifractal approach [7], the minimal spanning tree [8, 9, $10]$, the Minkowski functionals $[11,12]$, or the $J$ function $[13,14]$ which is defined as the ratio $J(r)=(1-$ $G(r)) /(1-F(r))$, where $F$ is the distribution function of the distance $r$ of an arbitrary point in $\mathbf{R}^{3}$ to the nearest object in the catalog, and $G$ is the distribution function of the distance $r$ of an object to the nearest object. Wavelets have also been used for analyzing the projected $2 \mathrm{D}$ or the $3 \mathrm{D}$ galaxy distribution $[15,16,17,18$, 19].

New geometric multiscale methods have recently emerged, the beamlet transform $[20,21]$ and the ridgelet transform [22]; these allow us to represent data containing, respectively, filaments and sheets, while wavelets represent well isotropic features (i.e., clusters in 3D). As each of these three transforms is tuned to a specific kind of feature, all of them are useful and should be combined to describe a given catalog.

Sections 2, 3, and 4 describe, respectively, the 3D wavelet transform, the $3 \mathrm{D}$ ridgelet transform, and the $3 \mathrm{D}$ beamlet transform. It is shown in Section 5 through a set of experiments how these three $3 \mathrm{D}$ transforms can be combined in order to describe statistically the distribution of galaxies.

\section{THE 3D WAVELET TRANSFORM}

\subsection{The undecimated isotropic wavelet transform}

For each $a>0, b_{1}, b_{2}, b_{3} \in \mathbf{R}^{3}$, the wavelet is defined by

$$
\begin{gathered}
\psi_{a, b_{1}, b_{2}, b_{3}}: \mathbf{R}^{3} \longrightarrow \mathbf{R}, \\
\psi_{a, b_{1}, b_{2}, b_{3}}\left(x_{1}, x_{2}, x_{3}\right)=a^{-3 / 2} \cdot \psi\left(\frac{x_{1}-b_{1}}{a}, \frac{x_{2}-b_{2}}{a}, \frac{x_{3}-b_{3}}{a}\right) .
\end{gathered}
$$

Given a function $f \in L^{2}\left(\mathbf{R}^{3}\right)$, we define its wavelet coefficients by

$$
\begin{gathered}
\mathcal{W}_{f}: \mathbf{R}^{4} \longrightarrow \mathbf{R}, \\
\mathcal{W}_{f}\left(a, b_{1}, b_{2}, b_{3}\right)=\int \bar{\psi}_{a, b_{1}, b_{2}, b_{3}}(\mathbf{x}) f(\mathbf{x}) d \mathbf{x} .
\end{gathered}
$$

Figure 1 shows an example of 3D wavelet function.

It is standard to digitize the transform for data $c(x, y, z)$ with $x, y, z \in\{1, \ldots, N\}$ as follows. The wavelet transform of a signal produces, at each scale $j$, a set of zero-mean coefficient values $\left\{w_{j}\right\}$. Let $\phi$ be a lowpass filter and we define $\phi_{j}(x)=\phi\left(2^{j} x\right)$ and $c_{j}=c * \phi_{j}$. Using an undecimated isotropic wavelet decomposition [23], the set $\left\{w_{j}\right\}$ has the same number of pixels as the signal and this wavelet transform is redundant. Furthermore, using a wavelet defined as the difference between the scaling functions of two successive scales

$$
\frac{1}{8} \psi\left(\frac{x}{2}, \frac{y}{2}, \frac{z}{2}\right)=\phi(x, y, z)-\frac{1}{8} \phi\left(\frac{x}{2}, \frac{y}{2}, \frac{z}{2}\right)
$$

the original cube $c=c_{0}$ can be expressed as the sum of all the wavelet scales and the smoothed array $c_{j}$ :

$$
c_{0, x, y, z}=c_{J, x, y, z}+\sum_{j=1}^{J} w_{j, x, y, z}
$$

The set $w=\left\{w_{1}, w_{2}, \ldots, w_{J}, c_{J}\right\}$ represents the wavelet transform of the data. If we let $W$ denote the wavelet transform operator and $N$ the pixels in $c$, the wavelet transform $w$ $\left(w=W_{c}\right)$ has $(J+1) N$ pixels, for a redundancy factor of $J+1$. The scaling function $\phi$ is generally chosen as a spline of degree 3 , and the $3 \mathrm{D}$ implementation is based on three $1 \mathrm{D}$ sets of (separable) convolutions. Like the scaling function $\phi$, the wavelet function $\psi$ is isotropic (see Figure 2). More details can be found in $[23,24]$.

\section{THE 3D RIDGELET TRANSFORM}

\subsection{The $2 \mathrm{D}$ ridgelet transform}

The $2 \mathrm{~d}$ continuous ridgelet transform of a function $f \in$ $L^{2}\left(\mathbf{R}^{2}\right)$ was defined in [22] as follows. 

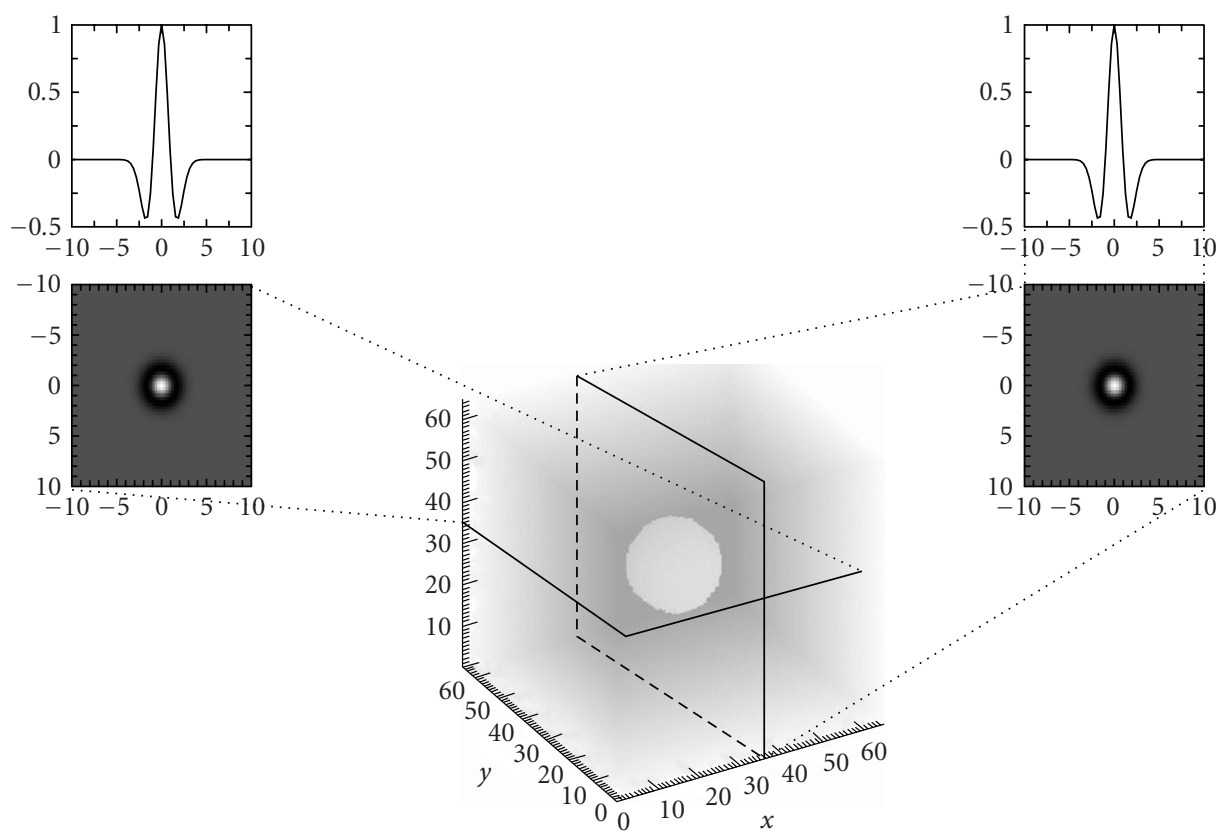

FIGURE 1: Example of wavelet function.

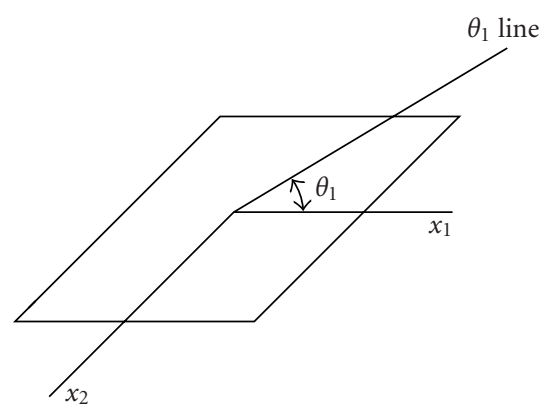

(a)

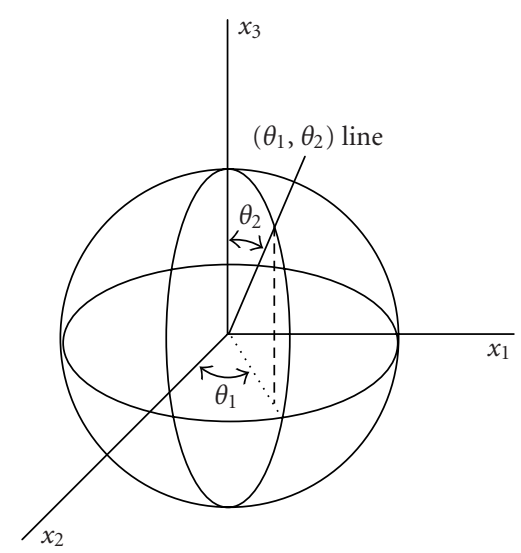

(b)

Figure 2: Definition of angle $1 \theta_{1}$ and $\theta_{2}$ in (a) $\mathbf{R}^{2}$ (2D case) and (b) $\mathbf{R}^{3}$ (3D case).

Select a smooth function $\psi \in L^{2}(\mathbf{R})$, satisfying admissibility condition

$$
\int \frac{|\hat{\psi}(\xi)|^{2}}{|\xi|} d \xi<\infty
$$

which holds if $\psi$ has a sufficient decay and a vanishing mean $\int \psi(t) d t=0$ ( $\psi$ can be normalized so that it has unit energy $\left.1 /(2 \pi) \int|\hat{\psi}(\xi)|^{2} d \xi=1\right)$. For each $a>0, b \in \mathbf{R}$, and $\theta_{1} \in$ $[0,2 \pi[$, we define the ridgelet by

$$
\begin{gathered}
\psi_{a, b, \theta_{1}}: \mathbf{R}^{2} \longrightarrow \mathbf{R} \\
\psi_{a, b, \theta_{1}}\left(x_{1}, x_{2}\right)=a^{-1 / 2} \cdot \psi\left(\frac{\left(x_{1} \cos \theta_{1}+x_{2} \sin \theta_{1}-b\right)}{a}\right) .
\end{gathered}
$$

Given a function $f \in L^{2}\left(\mathbf{R}^{2}\right)$, we define its ridgelet coefficients by

$$
\begin{gathered}
\mathcal{R}_{f}: \mathbf{R}^{3} \longrightarrow \mathbf{R}, \\
\mathcal{R}_{f}\left(a, b, \theta_{1}\right)=\int \bar{\psi}_{a, b, \theta_{1}}(\mathbf{x}) f(\mathbf{x}) d \mathbf{x} .
\end{gathered}
$$

It has been shown [22] that the ridgelet transform is precisely the application of a $1 \mathrm{D}$ wavelet transform to the slices of the Radon transform (where the angular variable $\theta_{1}$ is constant). This method is in a sense optimal to detect lines of the size of the image (the integration increase as the length of the line). More details on the implementation of the digital ridgelet transform can be found in [25]. 


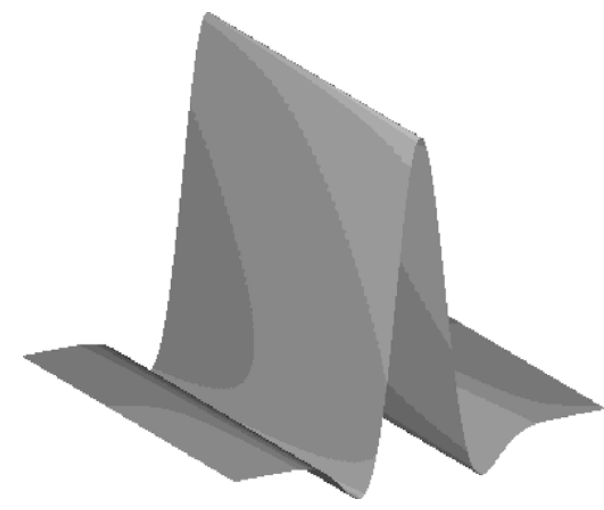

(a)

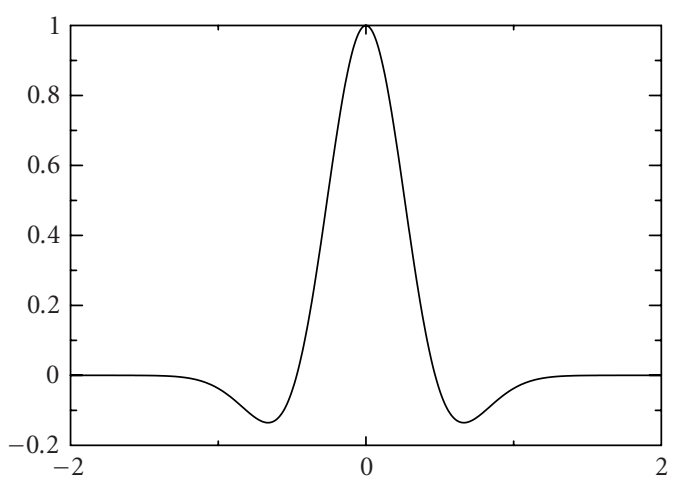

(b)

FIGURE 3: Example of 2D ridgelet function.

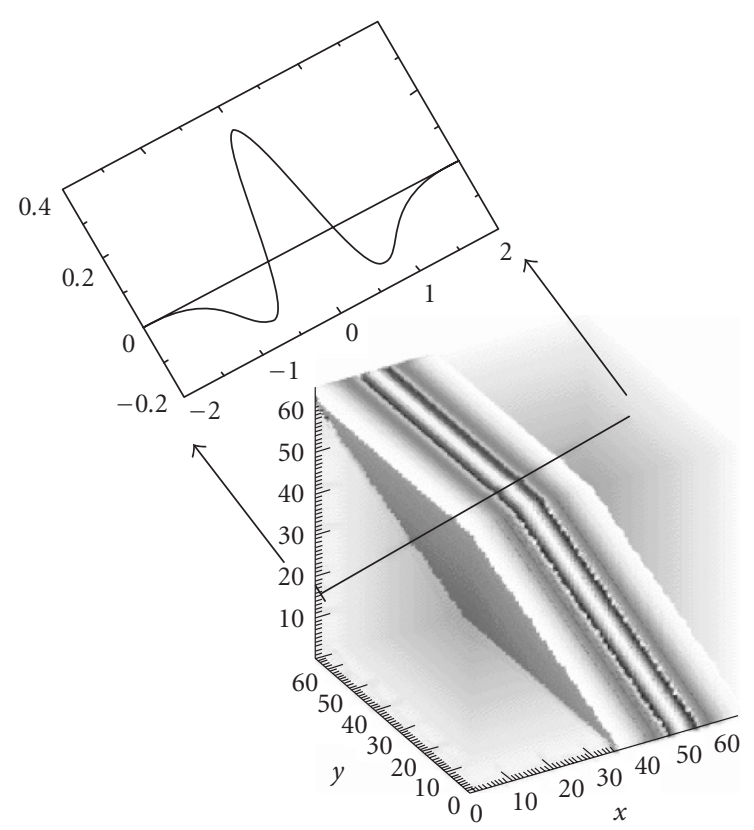

FIgURE 4: Example of ridgelet function.

Figure 3 (left) shows an example ridgelet function. This function is constant along lines $x_{1} \cos \theta+x_{2} \sin \theta=$ const. Transverse to these ridges it is a wavelet (see Figure 3(b)).

\subsection{From $2 D$ to $3 D$}

The 3D continuous ridgelet transform of a function $f \in$ $L^{2}\left(\mathbf{R}^{3}\right)$ is given by

$$
\begin{gathered}
\mathcal{R}_{f}: \mathbf{R}^{4} \longrightarrow \mathbf{R}, \\
\mathcal{R}_{f}\left(a, b, \theta_{1}, \theta_{2}\right)=\int \bar{\psi}_{a, b, \theta_{1}, \theta_{2}}(\mathbf{x}) f(\mathbf{x}) d \mathbf{x},
\end{gathered}
$$

(1) 3D-FFT. Compute $\hat{c}\left(k_{1}, k_{2}, k_{3}\right)$, the 3D FFT of the cube $c\left(i_{1}, i_{2}, i_{3}\right)$.

(2) Cartesian-to-spherical conversion. Using an interpolation scheme, substitute the sampled values of $\hat{c}$ obtained on the Cartesian coordinate system $\left(k_{1}, k_{2}, k_{3}\right)$ with sampled values in a spherical coordinate system $\left(\theta_{1}, \theta_{2}, \rho\right)$.

(3) Extract lines. Extract the $3 N^{2}$ lines (of size $N$ ) passing through the origin and the boundary of $\hat{c}$.

(4) $1 D$-IFFT. Compute the 1D inverse FFT on each line.

(5) $1 D-W T$. Compute the $1 \mathrm{D}$ wavelet transform on each line.

Algorithm 1: The 3D ridgelet transform algorithm.

where $a>0, b \in \mathbf{R}, \theta_{1} \in\left[0,2 \pi\left[\right.\right.$, and $\theta_{2} \in[0, \pi[$. The ridgelet function is defined by

$$
\begin{gathered}
\psi_{a, b, \theta_{1}, \theta_{2}}: \mathbf{R}^{3} \longrightarrow \mathbf{R}, \\
\psi_{a, b, \theta_{1}, \theta_{2}}\left(x_{1}, x_{2}, x_{3}\right) \\
=a^{-1 / 2} \cdot \psi\left(\frac{\left(x_{1} \cos \theta_{1} \cos \theta_{2}+x_{2} \sin \theta_{1} \cos \theta_{2}+x_{3} \sin \theta_{2}-b\right)}{a}\right) .
\end{gathered}
$$

Figure 4 shows an example of ridgelet function. It is a wavelet function in the direction defined by the line $\left(\theta_{1}, \theta_{2}\right)$, and it is constant along the orthogonal plane to this line.

As in the $2 \mathrm{D}$ case, the $3 \mathrm{D}$ ridgelet transform can be built by extracting lines in the Fourier domain. Let $c\left(i_{1}, i_{2}, i_{3}\right)$ be a cube of size $(N, N, N)$; the steps can be seen in Algorithm 1 steps.

Figure 5 shows the $3 \mathrm{D}$ ridgelet transform flowgraph. The $3 \mathrm{D}$ ridgelet transform allows us to detect sheets in a cube.

\section{Local 3D ridgelet transform}

The ridgelet transform is optimal to find sheets of the size of the cube. To detect smaller sheets, a partitioning must be 


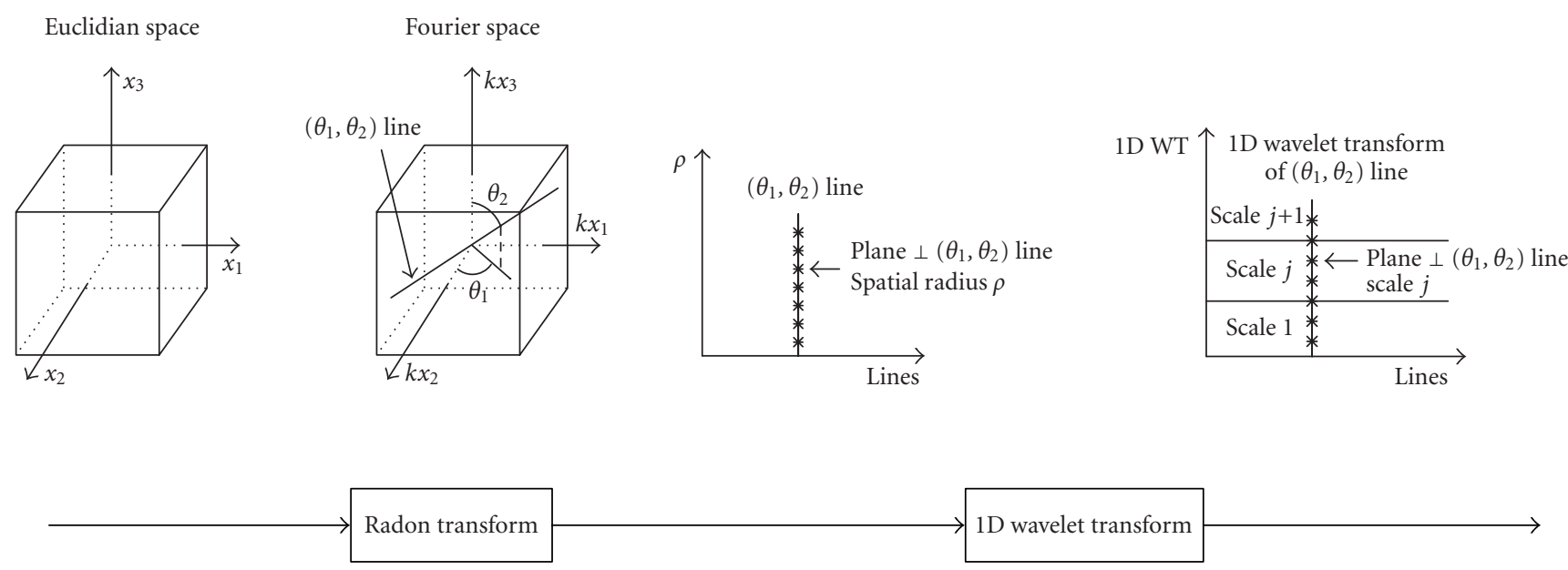

FIgURE 5: 3D ridgelet transform flowgraph.

introduced [26]. The cube $c$ is decomposed into blocks of lower side-length $b$ so that for a $N \times N \times N$ cube, we count $N / b$ blocks in each direction. After the block partitioning, the tranform is tuned for sheets of size $b \times b$ and of thickness $a_{j}$, $a_{j}$ corresponding to the different dyadic scales used in the transformation.

\section{THE 3D BEAMLET TRANSFORM}

\subsection{Definition}

The X-ray transform of a continuum function $f(x, y, z)$ with $(x, y, z) \in \mathbf{R}^{3}$ is defined by

$$
(X f)(L)=\int_{L} f(p) d p
$$

where $L$ is a line in $\mathbf{R}^{3}$, and $p$ is a variable indexing points in the line. The transformation contains all line integrals of $f$. The beamlet transform (BT) can be seen as a multiscale digital X-ray transform. It is multiscale transform because, in addition to the multiorientation and multilocation line integral calculation, it integrated also over line segments at different lengths. The 3D BT is an extension to the 2D BT, proposed by Donoho and Huo [20].

\section{The system of $3 D$ beams}

The transform requires an expressive set of line segments, including line segments with various lengths, locations, and orientations lying inside a 3D volume.

A seemingly natural candidate for the set of line segments is the family of all line segments between each voxel corner and every other voxel corner, the set of $3 D$ beams. For a 3D data set with $n^{3}$ voxels, there are $O\left(n^{6}\right) 3 \mathrm{D}$ beams. It is infeasible to use the collection of $3 \mathrm{D}$ beams as a basic data structure since any algorithm based on this set will have a complexity with lower bound of $n^{6}$ and hence be unworkable for typical 3D data size.

\subsection{The beamlet system}

A dyadic cube $C\left(k_{1}, k_{2}, k_{3}, j\right) \subset[0,1]^{3}$ is the collection of $3 \mathrm{D}$ points

$$
\begin{aligned}
\left\{\left(x_{1}, x_{2}, x_{3}\right):\right. & {\left[\frac{k_{1}}{2^{j}}, \frac{\left(k_{1}+1\right)}{2^{j}}\right] \times\left[\frac{k_{2}}{2^{j}}, \frac{\left(k_{2}+1\right)}{2^{j}}\right] } \\
& \left.\times\left[\frac{k_{3}}{2^{j}}, \frac{\left(k_{3}+1\right)}{2^{j}}\right]\right\},
\end{aligned}
$$

where $0 \leq k_{1}, k_{2}, k_{3}<2^{j}$ for an integer $j \geq 0$, called the scale.

Such cubes can be viewed as descended from the unit cube $C(0,0,0,0)=[0,1]^{3}$ by recursive partitioning. Hence, the result of splitting $C(0,0,0,0)$ in half along each axis is the eight cubes $C\left(k_{1}, k_{2}, k_{3}, 1\right)$ where $k_{i} \in\{0,1\}$ (see Figure 6), splitting those in half along each axis we get the 64 subcubes $C\left(k_{1}, k_{2}, k_{3}, 2\right)$ where $k_{i} \in\{0,1,2,3\}$, and if we decompose the unit cube into $n^{3}$ voxels using a uniform $n$-by- $n$-by- $n$ grid with $n=2^{J}$ dyadic, then the individual voxels are the $n^{3}$ cells $C\left(k_{1}, k_{2}, k_{3}, J\right), 0 \leq k_{1}, k_{2}, k_{3}<n$.

Associated to each dyadic cube we can build a system of line segments that have both of their end-points lying on the cube boundary. We call each such segment $a$ beamlet. If we consider all pairs of boundary voxel corners, we get $O\left(n^{4}\right)$ beamlets for a dyadic cube with a side-length of $n$ voxels (we actually work with a slightly different system in which each line is parametrized by a slope and an intercept instead of its end-points as explained below). However, we will still have $O\left(n^{4}\right)$ cardinality. Assuming a voxel size of $1 / n$ we get $J+1$ scales of dyadic cubes where $n=2^{J}$, for any scale $0 \leq j \leq J$ there are $2^{3 j}$ dyadic cubes of scale $j$ and since each 


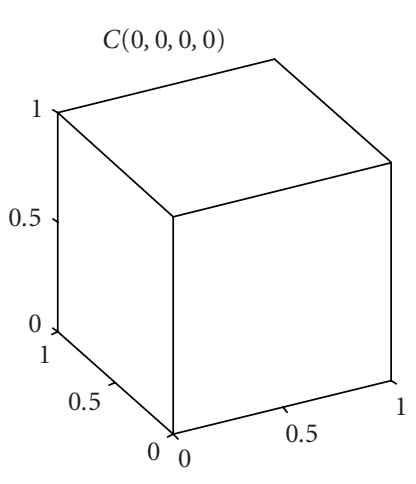

(a)

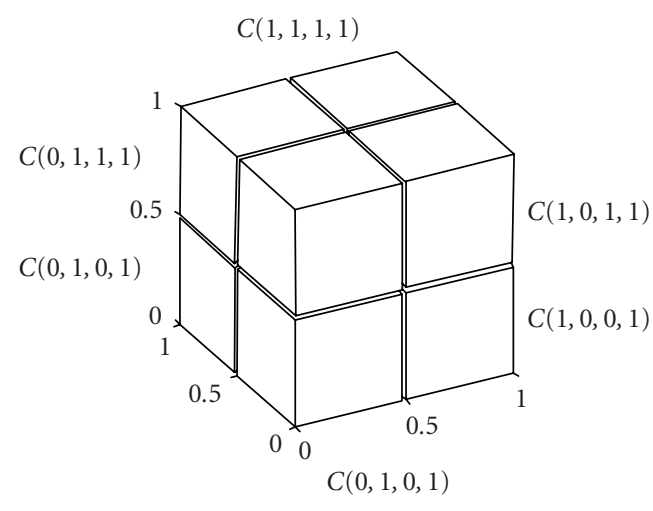

(b)

Figure 6: Dyadic cubes.

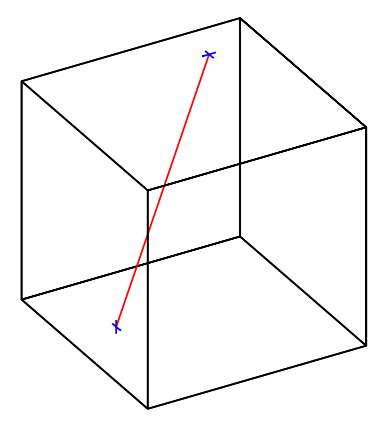

(a)

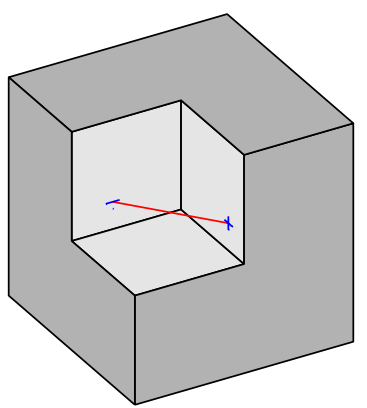

(b)

Figure 7: Examples of beamlets at two different scales: (a) scale 0 (coarsest scale) and (b) scale 1 (next finer scale).

dyadic cube at scale $j$ has a side-length of $2^{J-j}$ voxels, we get $O\left(2^{4(J-j)}\right)$ beamlets associated with the dyadic cube and a total of $O\left(2^{4 J-j}\right)=O\left(n^{4} / 2^{j}\right)$ beamlets at scale $j$. If we sum the number of beamlets at all scales we get $O\left(n^{4}\right)$ beamlets.

This gives a multiscale arrangement of line segments in $3 \mathrm{D}$ with controlled cardinality of $O\left(n^{4}\right)$, the scale of a beamlet is defined as the scale of the dyadic cube it belongs to so lower scales correspond to longer line segments and finer scales correspond to shorter line segments. Figure 7 shows 2 beamlets at different scales.

To index the beamlets in a given dyadic cube, we use slope-intercept coordinates. For a data cube of $n \times n \times n$ voxels, consider a coordinate system with the cube center of mass at the origin and a unit length for a voxel. Hence, for $(x, y, z)$ in the data cube we have $|x|,|y|,|z| \leq n / 2$. We can consider three kinds of lines: $x$-driven, $y$-driven, and $z$-driven, depending on which axis provides the shallowest slopes. An $x$-driven line takes the form

$$
z=s_{z} x+t_{z}, \quad y=s_{y} x+t_{y}
$$

with slopes $s_{z}, s_{y}$, and intercepts $t_{z}$ and $t_{y}$. Here the slopes $\left|s_{z}\right|,\left|s_{y}\right| \leq 1 . y$ - and $z$-driven lines are defined with an interchange of roles between $x$ and $y$ or $z$, as the case may be.
The slopes and intercepts run through equispaced sets:

$$
\begin{gathered}
s_{x}, s_{y}, s_{z} \in\left\{\frac{2 \ell}{n}: \ell=-\frac{n}{2}, \ldots, \frac{n}{2-1}\right\}, \\
t_{x}, t_{y}, t_{z} \in\left\{\ell:-\frac{n}{2}, \ldots, \frac{n}{2-1}\right\} .
\end{gathered}
$$

Beamlets in a data cube of side $n$ have lengths between $n / 2$ and $\sqrt{3} n$ (the main diagonal).

\section{Computational aspects}

Beamlet coefficients are line integrals over the set of beamlets. A digital 3D image can be regarded as a 3D piece-wise constant function and each line integral is just a weighted sum of the voxel intensities along the corresponding line segment. Donoho and Levi [21] discuss in detail different approaches for computing line integrals in a $3 \mathrm{D}$ digital image. Computing the beamlet coefficients for real application data sets can be a challenging computational task since for a data cube with $n \times n \times n$ voxels, we have to compute $O\left(n^{4}\right)$ coefficients. By developing efficient cache aware algorithms we are able to handle 3D data sets of size up to $n=256$ on a typical desktop computer in less than a day running time. 


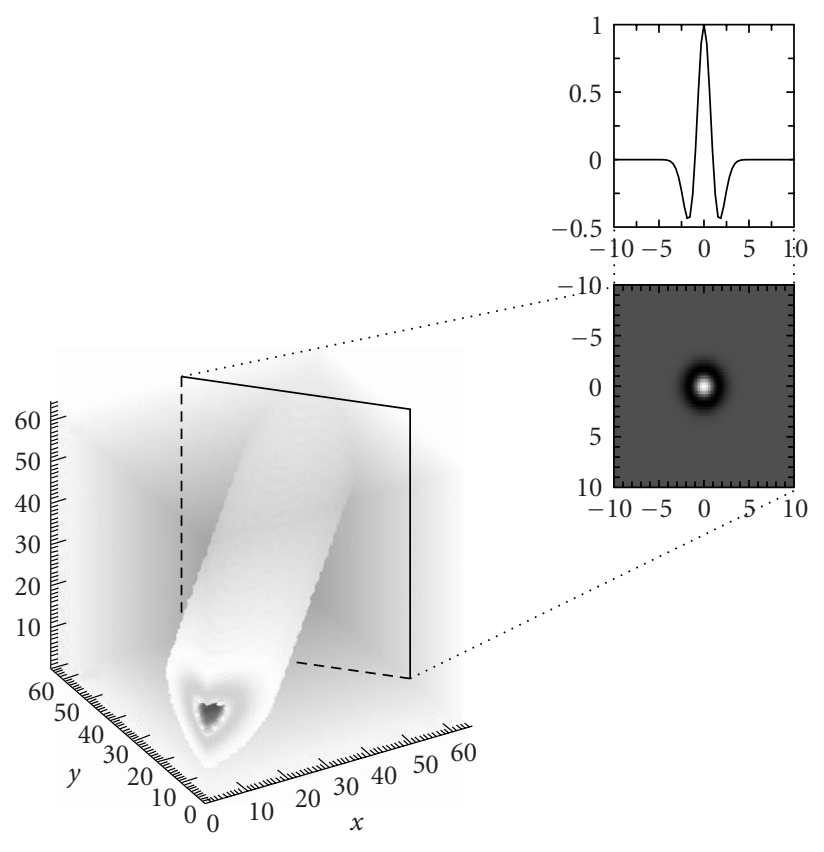

Figure 8: Example of beamlet function.

We will mention that in many cases there is no interest in the coarsest scales coefficient that consumes most of the computation time and in that case the overall running time can be significantly faster. The algorithms can also be easily implemented on a parallel machine of a computer cluster using a system such as MPI in order to solve bigger problems.
(1) $3 D$-FFT. Compute $\hat{c}\left(k_{1}, k_{2}, k_{3}\right)$, the three-dimensional FFT of the cube $c\left(i_{1}, i_{2}, i_{3}\right)$.

(2) Cartesian to spherical conversion. Using an interpolation scheme, substitute the sampled values of $\hat{c}$ obtained on the Cartesian coordinate system $\left(k_{1}, k_{2}, k_{3}\right)$ with sampled values in a spherical coordinate system $\left(\theta_{1}, \theta_{2}, \rho\right)$.

(3) Extract planes. Extract the $3 N^{2}$ planes (of size $N \times N$ ) passing through the origin (each line used in the $3 \mathrm{D}$ ridgelet transform defines a set of orthogonal planes; we take the one including the origin).

(4) 2D-IFFT. Compute the 2D inverse FFT on each plane.

(5) $2 \mathrm{D}-W T$. Compute the $2 \mathrm{D}$ wavelet transform on each plane.

Algorithm 2: The 3D beamlet transform algorithm.

\subsection{The FFT-based transformation}

Let $\psi \in L^{2}\left(\mathbf{R}^{2}\right)$ be a smooth function satisfying a $2 \mathrm{D}$ variant of the admissibility condition, the 3D continuous beamlet transform of a function $f \in L^{2}\left(\mathbf{R}^{3}\right)$ is given by

$$
\begin{gathered}
\mathcal{B}_{f}: \mathbf{R}^{5} \longrightarrow \mathbf{R}, \\
\mathcal{B}_{f}\left(a, b_{1}, b_{2}, \theta_{1}, \theta_{2}\right)=\int \bar{\psi}_{a, b, \theta_{1}, \theta_{2}}(\mathbf{x}) f(\mathbf{x}) d \mathbf{x},
\end{gathered}
$$

where $a>0, b_{1}, b_{2} \in \mathbf{R}, \theta_{1} \in\left[0,2 \pi\left[\right.\right.$, and $\theta_{2} \in[0, \pi[$. The beamlet function is defined by

$$
\begin{gathered}
\psi_{a, b_{1}, b_{2}, \theta_{1}, \theta_{2}}: \mathbf{R}^{3} \longrightarrow \mathbf{R}, \\
\psi_{a, b_{1}, b_{2}, \theta_{1}, \theta_{2}}\left(x_{1}, x_{2}, x_{3}\right)=a^{-1 / 2} \cdot \psi\left(\frac{\left(-x_{1} \sin \theta_{1}+x_{2} \cos \theta_{1}+b_{1}\right)}{a}, \frac{\left(x_{1} \cos \theta_{1} \cos \theta_{2}+x_{2} \sin \theta_{1} \cos \theta_{2}-x_{3} \sin \theta_{2}+b_{2}\right)}{a}\right) .
\end{gathered}
$$

Figure 8 shows an example of beamlet function. It is constant along lines of direction $\left(\theta_{1}, \theta_{2}\right)$, and a $2 \mathrm{D}$ wavelet function along plane orthogonal to this direction.

The 3D beamlet transform can be built using the "generalized projection-slice theorem" [27]. Let $f(\mathbf{x})$ be a function on $\mathbf{R}^{n}$; and let $\mathcal{R a d}_{m} f$ denote the $m$-dimensional partial Radon transform along the first $m$ directions, $m<n$. $\mathcal{R a d}_{m} f$ is a function of $\left(p, \mu_{m} ; x_{m+1}, \ldots, x_{n}\right), \boldsymbol{\mu}_{m}$ a unit directional vector in $\mathbf{R}^{n}$ (note that for a given projection angle, the $m$-dimensional partial Radon transform of $f(\mathbf{x})$ has $(n-m)$ untransformated spatial dimensions and a $(n-m+1)$ dimensional projection profile). In addition, let $\{\mathcal{F} f\}(\mathbf{k})$ denote the $n$-dimensional Fourier transform where $\mathbf{x}$ and $\mathbf{k}$ are conjugate variables.
The Fourier transform of the $m$-dimensional partial radon transform $\mathcal{R a d}_{m} f$ is related to the Fourier transform of $f(\mathcal{F} f)$ by the projection-slice relation

$$
\begin{array}{r}
\left\{\mathcal{F}_{n-m+1} \mathcal{R a d}_{m} f\right\}\left(k, k_{m+1}, \ldots, k_{n}\right) \\
=\{\mathcal{F} f\}\left(k \mu_{m}, k_{m+1}, \ldots, k_{n}\right) .
\end{array}
$$

Let $c\left(i_{1}, i_{2}, i_{3}\right)$ be a cube of size $(N, N, N)$; the steps of the Beamlet algorithm can be seen in the following Algorithm 2.

Figure 9 gives the 3D beamlet transform flowgraph. The 3D beamlet transform allows us to detect filaments in a cube. The beamlet transform algorithm presented in this section differs from the one presented in [28]; see the discussion in [21]. 


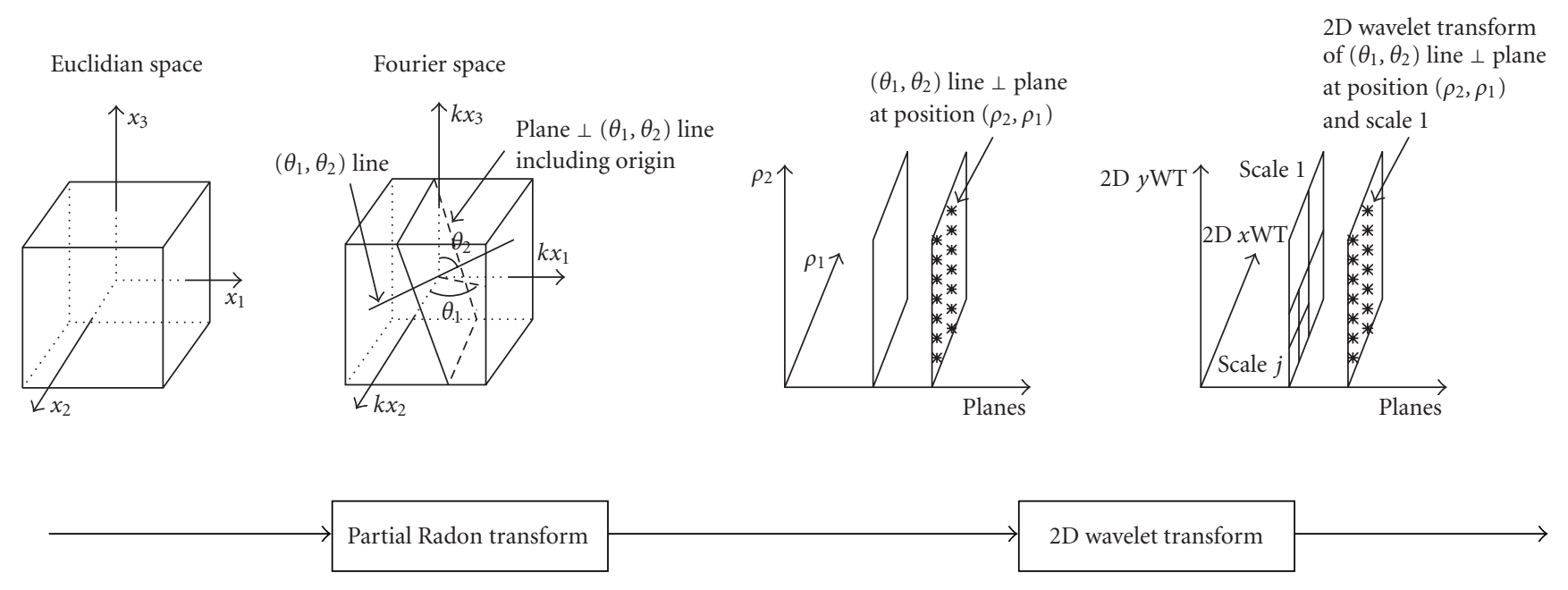

Figure 9: 3D beamlet transform flowgraph.

\section{EXPERIMENTS}

\subsection{Experiment 1}

We have simulated three data sets containing, respectively, a cluster, a plane, and a line. To each data set, Poisson noise has been added with eight different background levels. We applied the three transforms on the 24 simulated data sets. The coefficient distribution from each transformation was normalized using twenty realizations of a Poisson noise having the same number of counts as in the data.

Figure 10 shows the maximum value of the normalized distribution versus the noise level for our three simulated data sets. As expected, wavelets, ridgelets, and beamlets are, respectively, the best for detecting clusters, sheets, and lines. A feature can typically be detected with a very high signal-to-noise ratio in a matched transform, while remaining indetectible in some other transforms. For example, the wall is detected at more than $60 \sigma$ by the ridgelet transform, but at less than $5 \sigma$ by the wavelet transform. The line is detected almost at $10 \sigma$ by the beamlet transform, and with worse than $3 \sigma$ detection level by wavelets. These results show the importance of using several transforms for an optimal detection of all features contained in a data set.

\subsection{Experiment 2}

We use here two simulated data sets to illustrate the discriminative power of multiscale methods. The first one is a simulation from stochastic geometry. It is based on a Voronoi model. The second one is a mock catalog of the galaxy distribution drawn from a $\Lambda$-CDM N-body cosmological model [29]. Both processes have very similar two-point correlation functions at small scales, although they look quite different and have been generated following completely different algorithms.

(i) The first comes from Voronoi simulation. We locate a point in each of the vertices of a Voronoi tessellation of 1500 cells defined by 1500 nuclei distributed following a binomial process. There are 10085 vertices lying within a box of $141.4 h^{-1} \mathrm{Mpc}$ side.

(ii) The second point pattern represents the galaxy positions extracted from a cosmological $\Lambda$-CDM Nbody simulation. The simulation has been carried out by the Virgo consortium and related groups (see http://www.mpa-garching.mpg.de/Virgo). The simulation is a low-density $(\Omega=0.3)$ model with cosmological constant $\Lambda=0.7$. It is, therefore, an approximation to the real galaxy distribution [29]. There are 15445 galaxies within a box with side $141.3 h^{-1} \mathrm{Mpc}$. Galaxies in this catalog have stellar masses exceeding $2 \times 10^{10} M_{\odot}$.

Figure 11 shows the two simulated data sets, and Figure 12 shows the two-point correlation function curve for the two-point processes. The two-point fields are different, but as can be seen in Figure 12, both have very similar two-point correlation functions in a huge range of scales (2 decades).

We have applied the three transforms to each data set, and we have calculated the skewness vector $S=\left(s_{w}^{j}, s_{r}^{j}, s_{b}^{j}\right)$ and the kurtosis vector $K=\left(k_{w}^{j}, k_{r}^{j}, k_{b}^{j}\right)$ at each scale $j$. $s_{w}^{j}, s_{r}^{j}, s_{b}^{j}$ are, respectively, the skewness at scale $j$ of the wavelet coefficients, the ridgelet coefficients, and the beamlet coefficients. $k_{w}^{j}, k_{r}^{j}, k_{b}^{j}$ are, respectively, the kurtosis at scale $j$ of the wavelet coefficients, the ridgelet coefficients, and the beamlet coefficients. Figure 13 shows the kurtosis and the skewness vectors of the two data sets at the two first scales. In contrast to the case with the two-point correlation function, this figure shows strong differences between the two data sets, particularly on the wavelet axis, which indicates that the second data contains more or higher density clusters than the first one. 

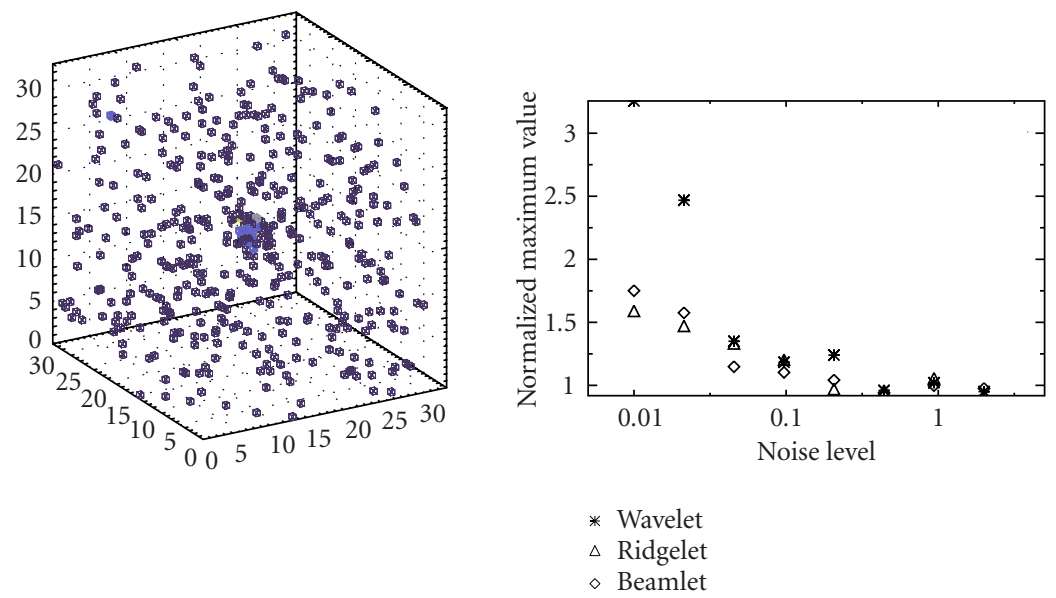

(a)
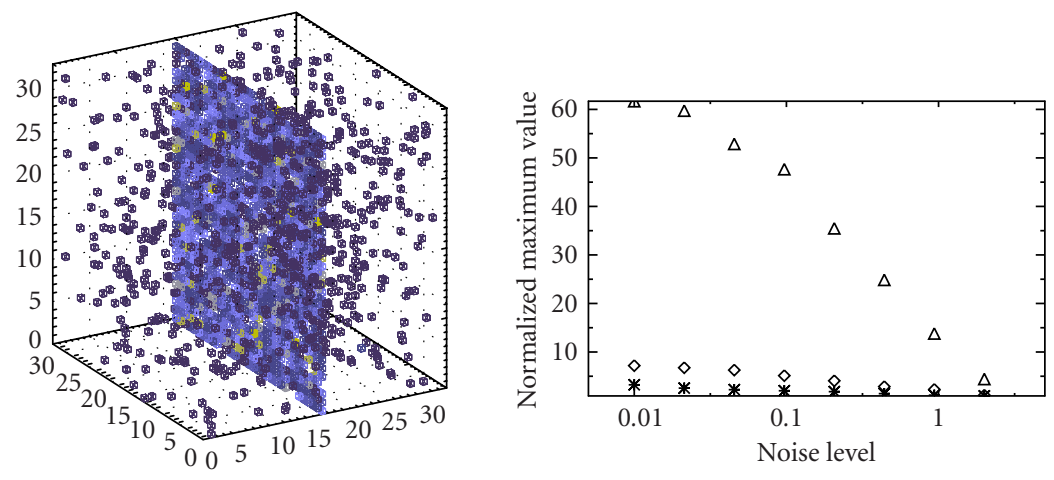

* Wavelet

$\triangle$ Ridgelet

$\diamond$ Beamlet

(b)
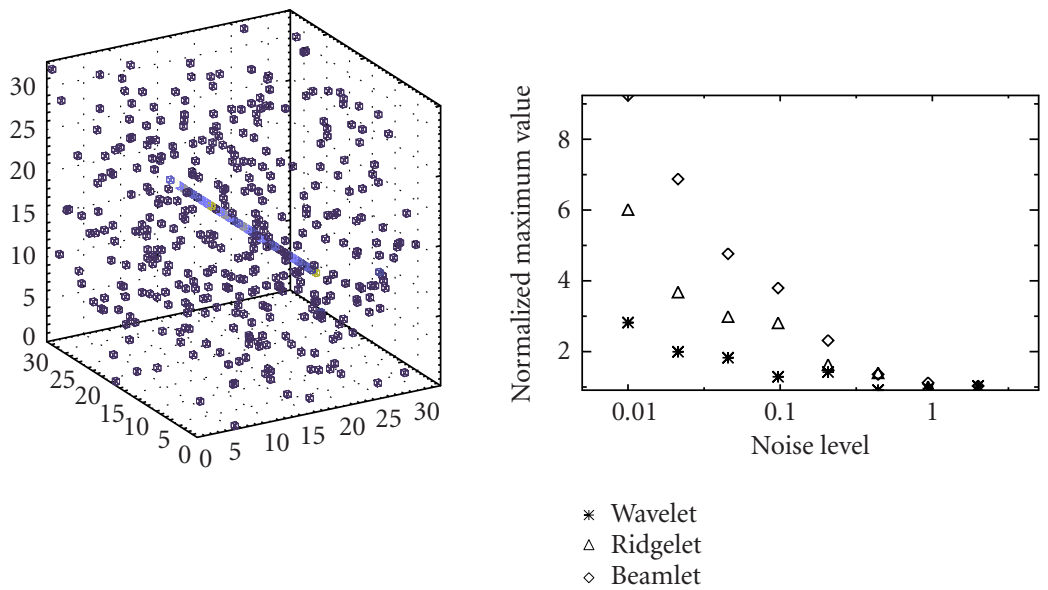

(c)

Figure 10: Poisson realization for a low noise level: simulation of cubes containing (a) a cluster, (b) a plane, and (c) a line. 


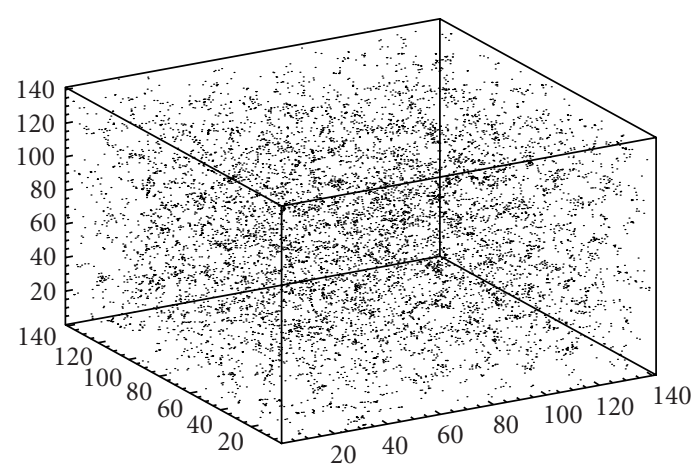

(a)

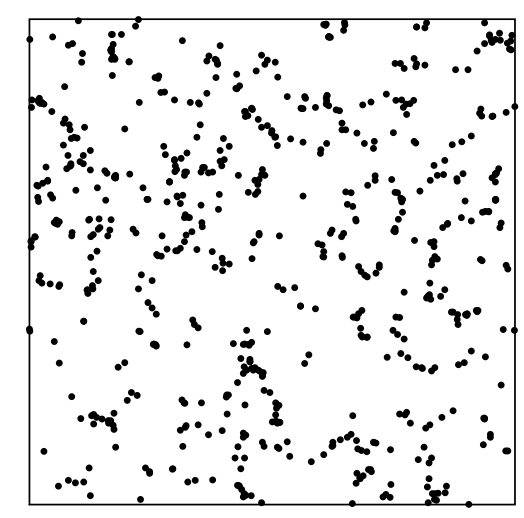

(c)

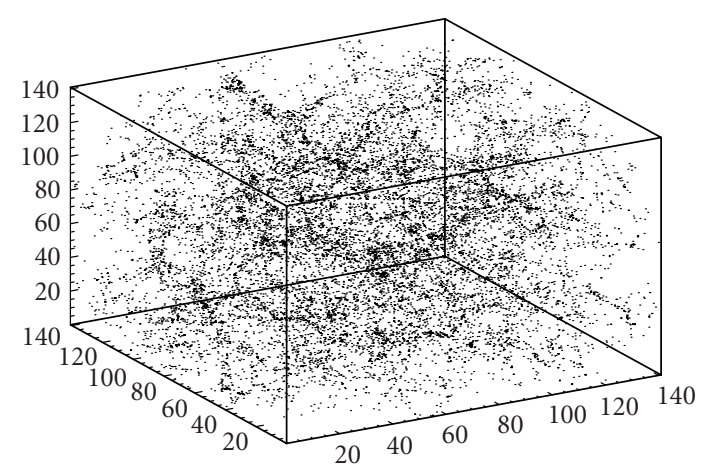

(b)

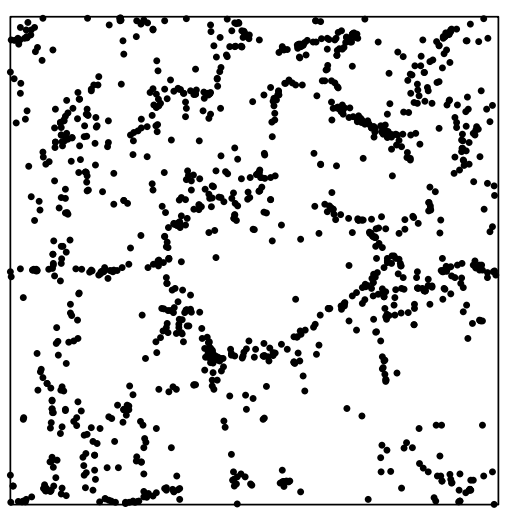

(d)

FIgURE 11: Simulated data sets. (a) The Voronoi vertices point pattern and (b) the galaxies of the GIF $\Lambda$-CDM N-body simulation. (c) One $10 \mathrm{~h}^{-1}$ width slice of each data set.

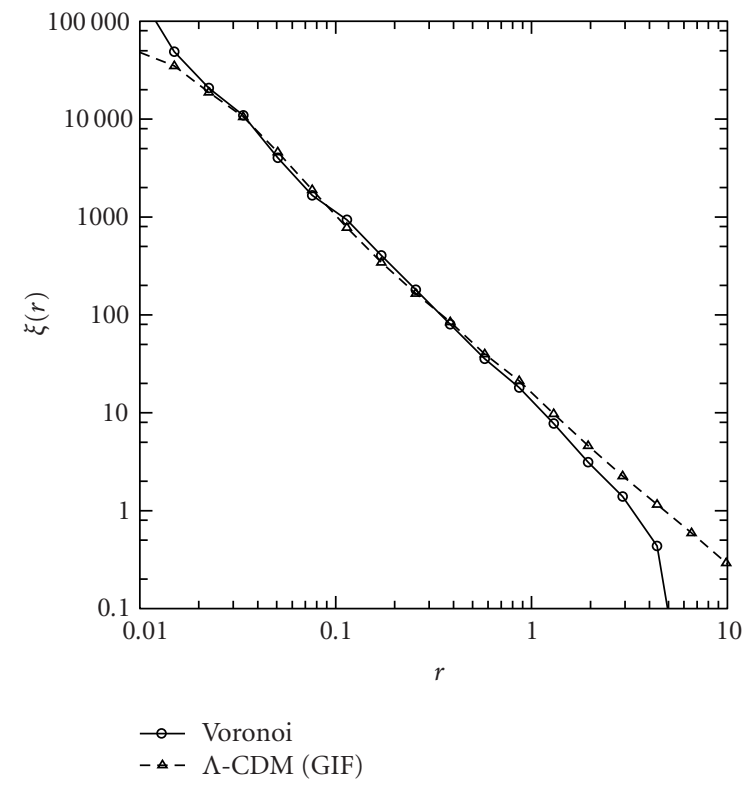

FIgURE 12: The two-point correlation function of the Voronoi vertices process and the GIF $\Lambda$-CDM N-body simulation. They are very similar in the range $[0.02,2] h^{-1} \mathrm{Mpc}$.

\subsection{Experiment 3}

In this experiment, we have used a $\Lambda$-CDM simulation based on the N-body hydrodynamical code, RAMSES [30]. The simulation uses an adaptive mesh refinement (AMR) technique, with a tree-based data structure allowing recursive grid refinements on a cell-by-cell basis. The simulated data were obtained using $256^{3}$ particles and $4.1 \times 10^{7}$ cells in the AMR grid, reaching a formal resolution of $8192^{3}$. The box size was set to $100 h^{-1} \mathrm{Mpc}$, with the following cosmological parameters:

$$
\begin{gathered}
\Omega_{m}=0.3, \quad \Omega_{\lambda}=0.7, \quad \Omega_{b}=0.039, \\
h=0.7, \quad \sigma_{8}=0.92 .
\end{gathered}
$$

We used the results of this simulation at six different redshifts $(z=5,3,2,1,0.5,0)$. Figure 14 shows a projection of the simulated cubes along one axis. We applied the $3 \mathrm{D}$ wavelet transform, the 3D beamlet transform, and the $3 \mathrm{D}$ ridgelet transform on the six data sets. Let $\sigma_{W, z, j}^{2}, \sigma_{R, z, j}^{2}$, $\sigma_{B, z, j}^{2}$ denote the variance of the wavelet, the ridgelet, and the beamlet coefficients of the scale $j$ at redshift $z$. 

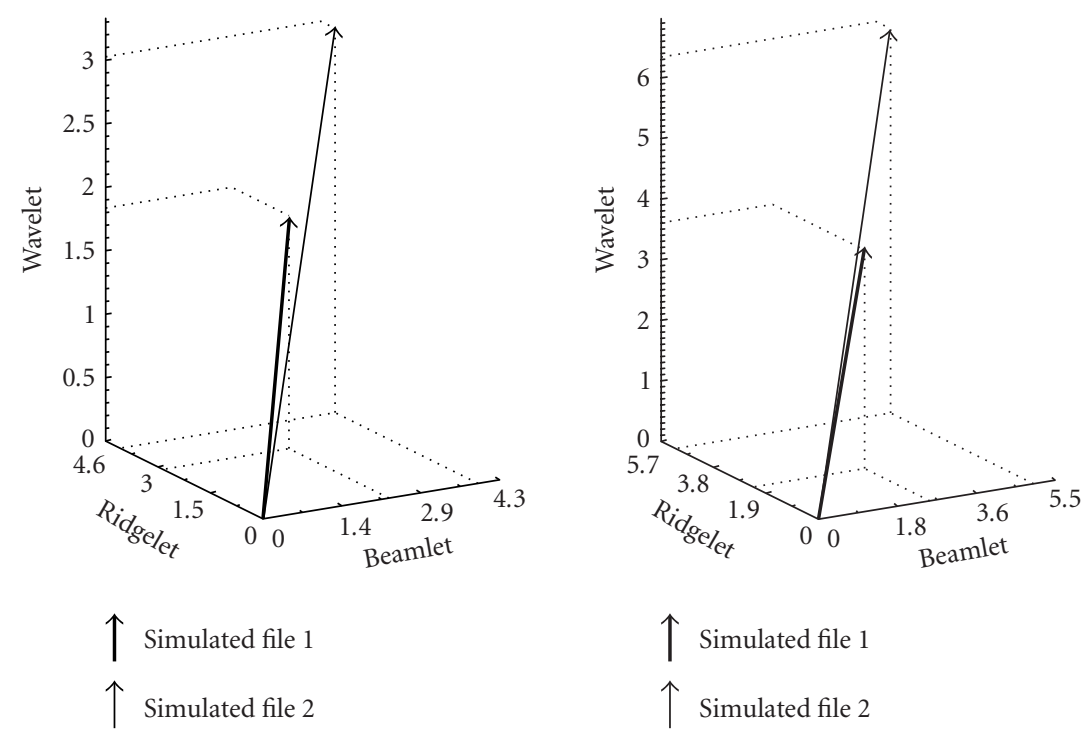

$\uparrow$ Simulated file 1
$\uparrow$ Simulated file 2

(a)

(b)
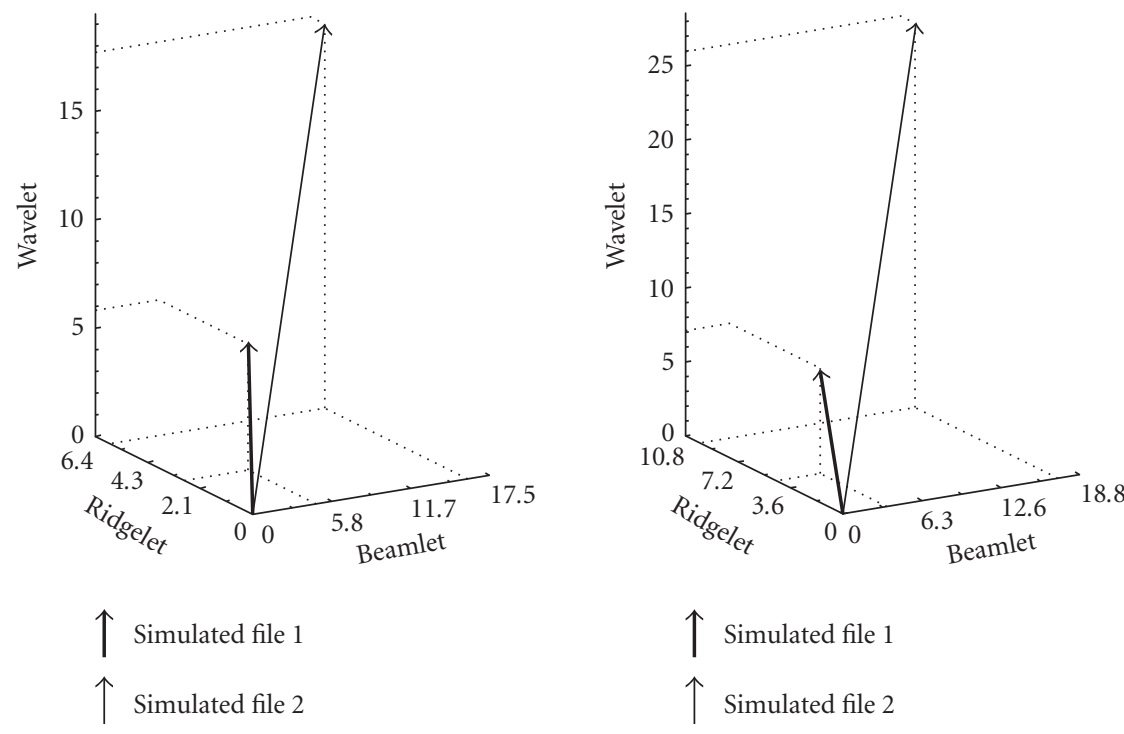

(c)

(d)

FIGURE 13: Skewness and kurtosis for the two simulated data sets: (a) skewness, scale 1, (b) skewness, scale 2, (c) kurtosis, scale 1, and (d) kurtosis, scale 2 .

Figure 15, shows, respectively, from top to bottom, the wavelet spectrum $P_{w}(z, j)=\sigma_{W, z, j}^{2}$, the beamlet spectrum $P_{b}(z, j)=\sigma_{B, z, j}^{2}$, and the ridgelet spectrum $P_{r}(z, j)=$ $\sigma_{R, z, j}^{2}$. In order to see the evolution of matter distribution with redshift and scale, we calculate the ratio $M_{w / b}(j, z)=$ $P_{w}(z, j) / P_{b}(z, j)$ and $M_{w / r}(j, z)=P_{w}(z, j) / P_{r}(z, j)$.

Figure 16 shows the $M_{w / b}$ and $M_{w / r}$ curves as a function of $z$ and Figure 17 shows the $M_{w / b}^{-1}$ and $M_{w / r}^{-1}$ curves as a function of the scale number $j$.
The $M_{w / b}$ curve does not show much evolution, while the $M_{w / r}$ curve presents a significant slope. This shows that the beamlet transform is more sensitive to clustering than the ridgelet transform. This is not surprising since the support of beamlets is much smaller than the support of ridgelets. $M_{w / r}$ increases with $z$, reflecting the cluster formation. The combination of multiscale transformations gives clear information about the degree of clustering, filamentarity, and sheetedness. 

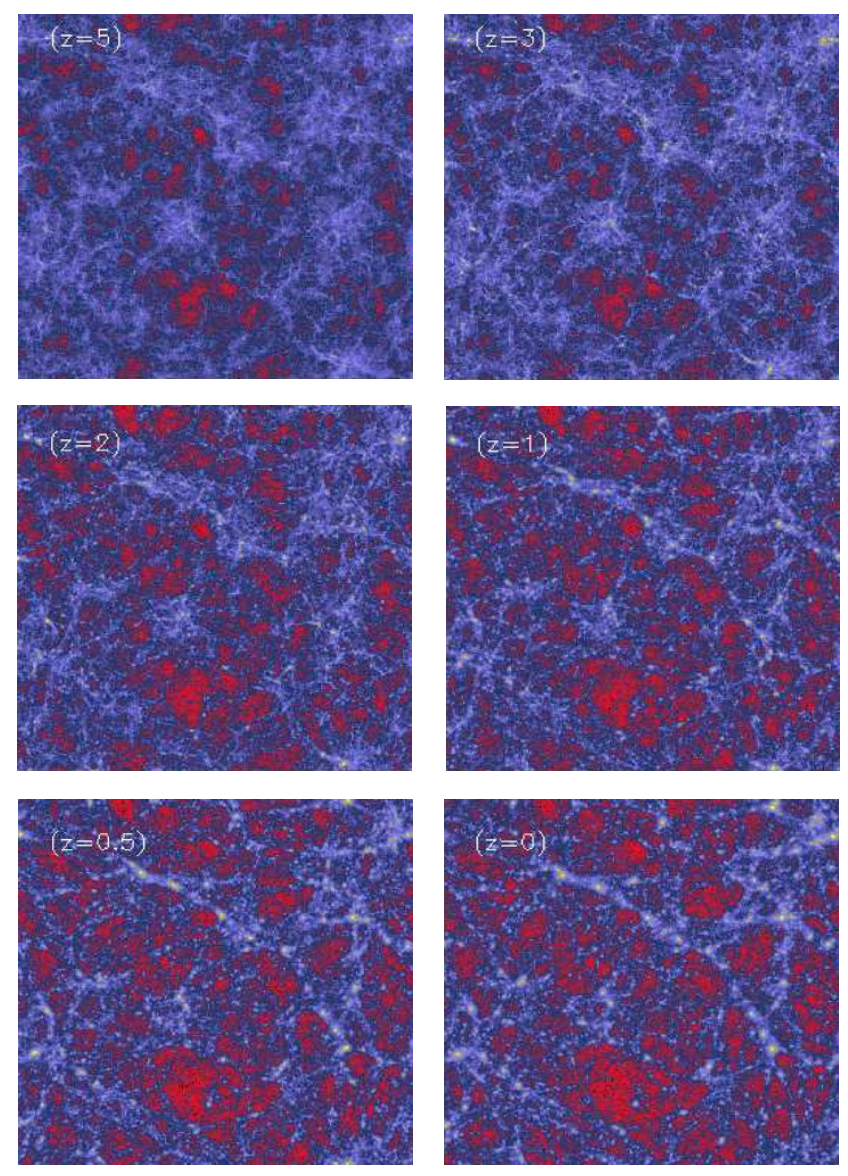

FIGURE 14: $\Lambda$-CDM simulation at different redshifts.

\section{CONCLUSION}

We have introduced in this paper a new method to analyze catalogs of galaxies based on the distribution of coefficients obtained by several geometric multiscale transforms.

We have introduced two new multiscale decompositions, the $3 \mathrm{D}$ ridgelet transform and the $3 \mathrm{D}$ beamlet transform, matched to sheetlike and filament features, respectively. We described fast implementations using FFTs. We showed that combining the information related to wavelet, ridgelet, and beamlet coefficients leads to a new description of point catalogs. In this paper, we described transform coefficients using skewness and kurtosis, but another recent statistic estimator such the higher criticism [31] could be used as well. Each multiscale transform is very sensitive to one kind of feature: wavelets to clusters; beamlets to filaments; and ridgelets to walls. A similar method has been proposed for analyzing CMB maps [32] where both the curvelet and the wavelet transforms were used for the detection and the discrimination of non-Gaussianities. This combined multiscale statistic is very powerful and we have shown that two data sets with identical two-point correlation functions are clearly distinguished by our approach. These new tools lead to better constraints on cosmological models.

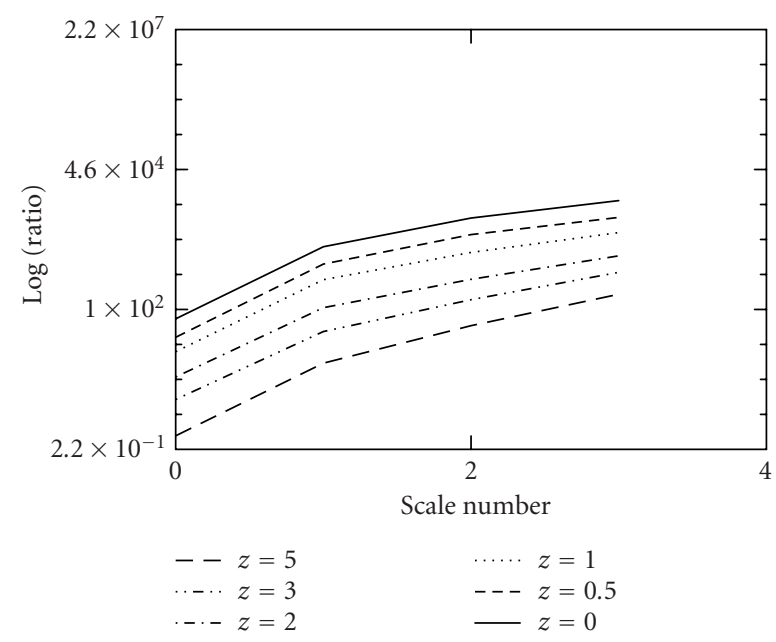

(a)
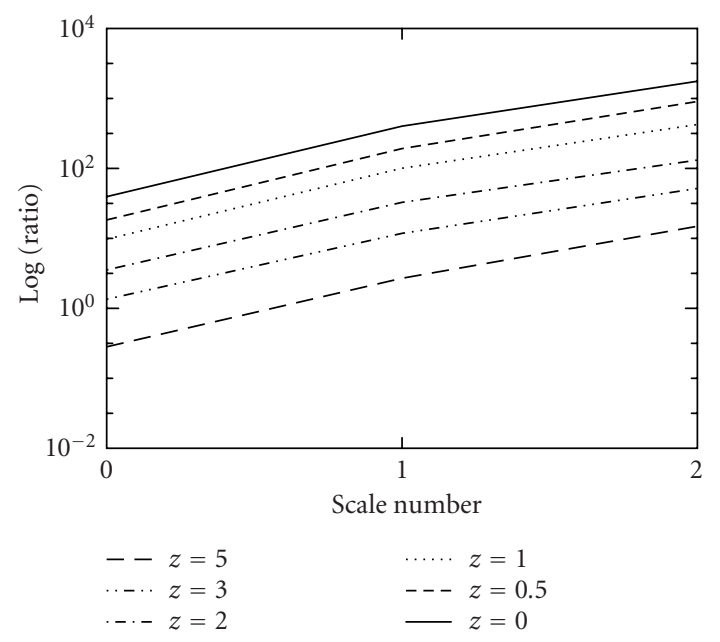

(b)

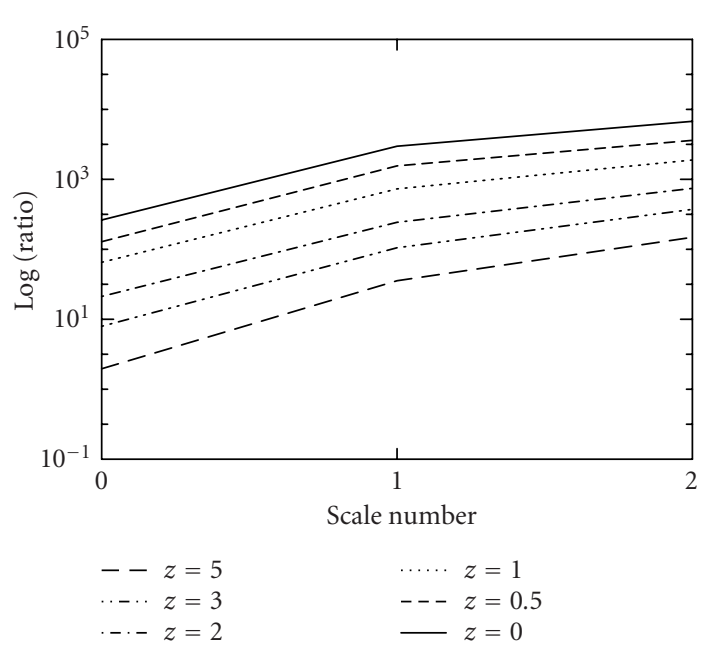

(c)

Figure 15: (a) Wavelet spectrum, (b) beamlet spectrum, and (c) ridgelet spectrum at different redshifts. 


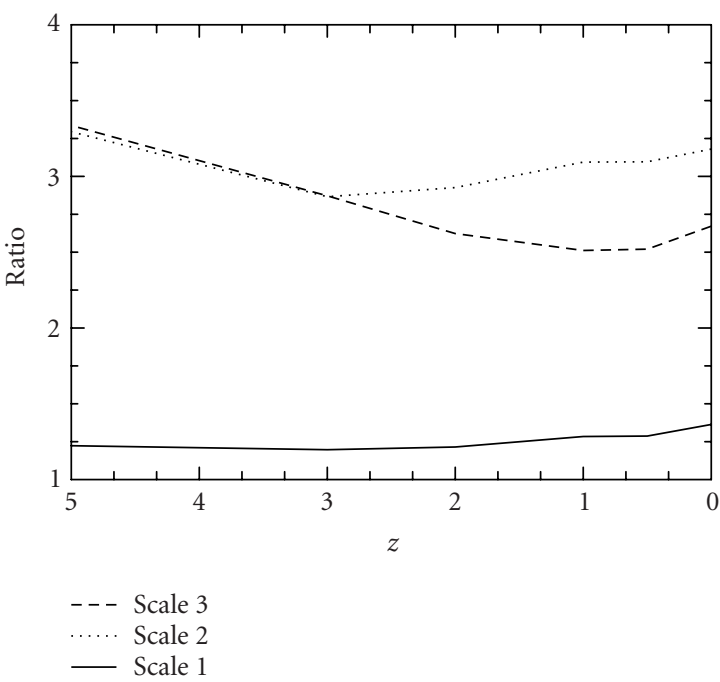

(a)

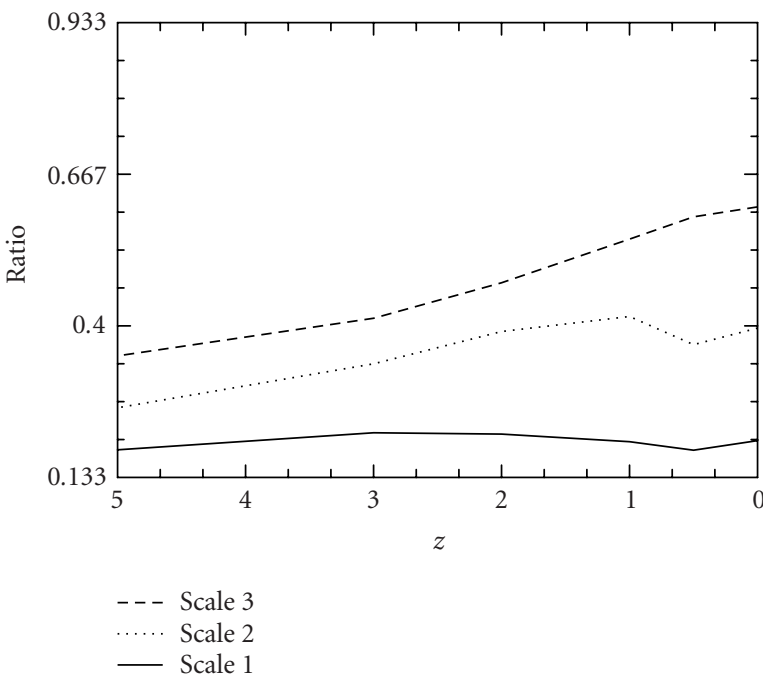

(b)

FIGURE 16: (a) Wavelet/beamlet $M_{w / b}(z, j)$ and (b) wavelet/ridgelet $M_{w / r}(z, j)$ curves for the scale number $j$ equal to 1,2 , and 3 .

\section{ACKNOWLEDGMENTS}

We wish to thank Romain Teyssier for giving us the $\Lambda$ CDM simulated data used in the third experiment. This work has been supported by the Spanish MCyT project AYA200308739-C02-01 (including FEDER), the Generalitat Valenciana project GRUPOS03/170, the National Science Foundation Grant DMS-01-40587 (FRG), and the Estonian Science Foundation Grant 4695.

\section{REFERENCES}

[1] P. J. E. Peebles, The Large-Scale Structure of the Universe, Princeton University Press, Princeton, NJ, USA, 1980.

[2] M. Tegmark, M. R. Blanton, M. A. Strauss, et al., "The threedimensional power spectrum of galaxies from the sloan digital

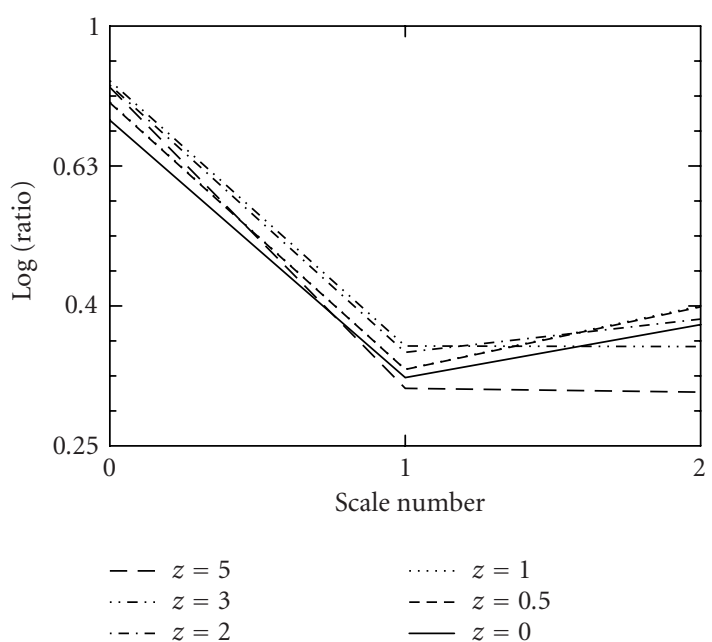

(a)

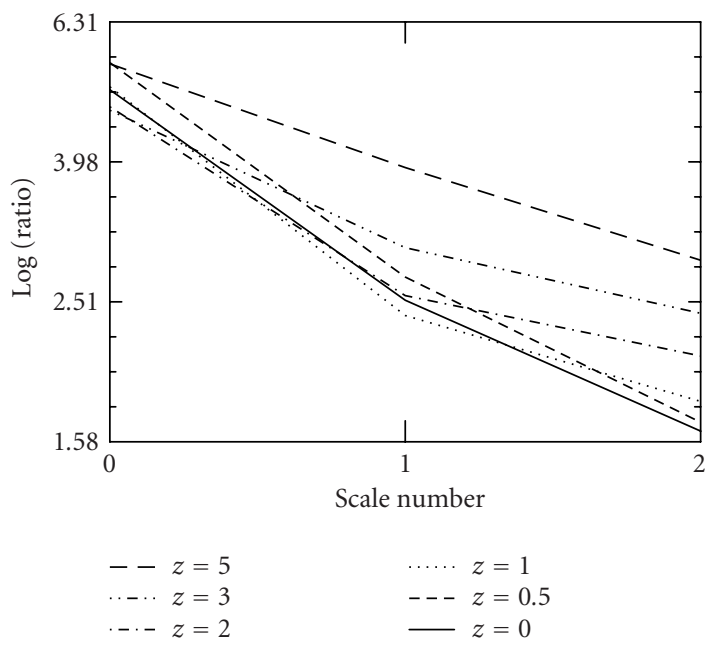

(b)

Figure 17: (a) Beamlet/wavelet $1 / M_{w / b}(z, j)$ and (b) ridgelet/ wavelet $1 / M_{w / r}(z, j)$ curves at different redshifts.

sky survey," The Astrophysical Journal, vol. 606, no. 2, part 1, pp. 702-740, 2004.

[3] S. Szapudi and A. S. Szalay, "A new class of estimators for the N-point correlations," Astrophysical Journal Letters, vol. 494, no. 1, pp. L41-L44, 1998.

[4] P. J. E. Peebles, "The galaxy and mass N-point correlation functions: a blast from the past," in Historical Development of Modern Cosmology, V. J. Martínez, V. Trimble, and M. J. PonsBordería, Eds., vol. 252 of ASP Conference Series, Astronomical Society of the Pacific, San Francisco, Calif, USA, 2001.

[5] V. J. Martínez and E. Saar, Statistics of the Galaxy Distribution, Chapman \& Hall/CRC press, Boca Raton, Fla, USA, 2002.

[6] S. Maurogordato and M. Lachieze-Rey, "Void probabilities in the galaxy distribution-scaling and luminosity segregation," The Astrophysical Journal, vol. 320, pp. 13-25, September 1987. 
[7] V. J. Martínez, B. J. T. Jones, R. Domínguez-Tenreiro, and R. van de Weygaert, "Clustering paradigms and multifractal measures," The Astrophysical Journal, vol. 357, no. 1, pp. 5061, 1990.

[8] S. P. Bhavsar and R. J. Splinter, "The superiority of the minimal spanning tree in percolation analyses of cosmological data sets," Monthly Notices of the Royal Astronomical Society, vol. 282, no. 4, pp. 1461-1466, 1996.

[9] L. G. Krzewina and W. C. Saslaw, "Minimal spanning tree statistics for the analysis of large-scale structure," Monthly Notices of the Royal Astronomical Society, vol. 278, no. 3, pp. 869876, 1996.

[10] A. G. Doroshkevich, D. L. Tucker, R. Fong, V. Turchaninov, and H. Lin, "Large-scale galaxy distribution in the Las Campanas Redshift Survey," Monthly Notices of the Royal Astronomical Society, vol. 322, no. 2, pp. 369-388, 2001.

[11] K. R. Mecke, T. Buchert, and H. Wagner, "Robust morphological measures for large-scale structure in the universe," Astronomy \& Astrophysics, vol. 288, no. 3, pp. 697-704, 1994.

[12] M. Kerscher, "Statistical analysis of large-scale structure in the universe," in Statistical Physics and Spatial Statistics: The Art of Analyzing and Modeling Spatial Structures and Pattern Formation, K. Mecke and D. Stoyan, Eds., vol. 554 of Lecture Notes in Physics, pp. 36-71, Springer, Berlin, Germany, 2000.

[13] M. N. M. Van Lieshout and A. J. Baddeley, "A nonparametric measure of spatial interaction in point patterns," Statistica Neerlandica, vol. 50, no. 3, pp. 344-361, 1996.

[14] M. Kerscher, M. J. Pons-Bordería, J. Schmalzing, et al., "A global descriptor of spatial pattern interaction in the galaxy distribution," The Astrophysical Journal, vol. 513, no. 2, part 1, pp. 543-548, 1999.

[15] E. Escalera, E. Slezak, and A. Mazure, "New evidence for subclustering in the Coma cluster using the wavelet analysis," Astronomy \& Astrophysics, vol. 264, no. 2, pp. 379-384, 1992.

[16] E. Slezak, V. de Lapparent, and A. Bijaoui, "Objective detection of voids and high-density structures in the first CfA redshift survey slice," The Astrophysical Journal, vol. 409, no. 2, pp. 517-529, 1993.

[17] V. J. Martínez, S. Paredes, and E. Saar, "Wavelet analysis of the multifractal character of the galaxy distribution," Monthly Notices of the Royal Astronomical Society, vol. 260, no. 2, pp. 365 375, 1993.

[18] A. Pagliaro, V. Antonuccio-Delogu, U. Becciani, and M. Gambera, "Substructure recovery by three-dimensional discrete wavelet transforms," Monthly Notices of the Royal Astronomical Society, vol. 310, no. 3, pp. 835-841, 1999.

[19] T. Kurokawa, M. Morikawa, and H. Mouri, "Scaling analysis of galaxy distribution in the Las Campanas Redshift Survey data," Astronomy \& Astrophysics, vol. 370, no. 2, pp. 358-364, 2001.

[20] D. L. Donoho and X. Huo, "Beamlets and multiscale image analysis," in Multiscale and Multiresolution Methods, vol. 20 of Lecture Notes in Computational Science and Engineering, pp. 149-196, Springer, New York, NY, USA, 2001.

[21] D. L. Donoho and O. Levi, "Fast x-ray and beamlet transforms for three-dimensional data," in Modern Signal Processing, D. Rockmore and D. Healy, Eds., vol. 46 of Mathematical Science Research Institute Publications, Cambridge University Press, Cambridge, UK, March 2002.

[22] E. J. Candès and D. L. Donoho, "Ridgelets: the key to high dimensional intermittency?" Philosophical Transactions of the Royal Society of London A, vol. 357, pp. 2495-2509, September 1999.

[23] J.-L. Starck, F. Murtagh, and A. Bijaoui, Image Processing and
Data Analysis: The Multiscale Approach, Cambridge University Press, Cambridge, UK, 1998.

[24] J.-L. Starck and F. Murtagh, "Astronomical Image and Data Analysis,” @\#pages, Springer, Berlin, Germany, 2002.

[25] J.-L. Starck, E. J. Candès, and D. L. Donoho, "The curvelet transform for image denoising," IEEE Trans. Image Processing, vol. 11, no. 6, pp. 670-684, 2002.

[26] E. J. Candès, "Harmonic analysis of neural networks," Applied and Computational Harmonic Analysis, vol. 6, no. 2, pp. 197218, 1999.

[27] P. C. Lauterbur and Z.-O. Liang, Principle of Magnetic Resonance Imaging, IEEE Press, New York, NY, USA, 2000.

[28] D. L. Donoho, O. Levi, J.-L. Starck, and V. J. Martínez, "Multiscale geometric analysis for 3D catalogs," in Astronomical Data Analysis II, J.-L. Starck and F. Murtagh, Eds., vol. 4847 of Proceedings of SPIE, pp. 101-111, Waikoloa, Hawaii, USA, August 2002.

[29] G. Kauffmann, J. M. Colberg, A. Diaferio, and S. D. M. White, "Clustering of galaxies in a hierarchical universe-I. Methods and results at $z=0$," Monthly Notices of the Royal Astronomical Society, vol. 303, no. 1, pp. 188-206, 1999.

[30] R. Teyssier, "Cosmological hydrodynamics with adaptive mesh refinement-A new high resolution code called RAMSES," Astronomy \& Astrophysics, vol. 385, no. 1, pp. 337-364, 2002.

[31] D. L. Donoho and J. Jin, "Higher criticism for detecting sparse heterogeneous mixtures," Tech. Rep., Statistics Department, Stanford University, Stanford, Calif, USA, 2002.

[32] J.-L. Starck, N. Aghanim, and O. Forni, "Detection and discrimination of cosmological non-Gaussian signatures by multi-scale methods," Astronomy \& Astrophysics, vol. 416, no. 1, pp. 9-17, 2004.

J-L. Starck has a Ph.D. degree from the University Nice-Sophia Antipolis and a Habilitation degree from the University Paris XI. He was a Visitor at the European Southern Observatory (ESO) in 1993, at UCLA in 2004, and at the Statistics Department, Stanford University, in 2000 and 2005. He has been a researcher at the service d'Astrophysique, CEA-sacloy, since 1994. His research interests include image processing, statistical methods in astrophysics, and cosmology. He is also author of two books entitled Image Processing and Data Analysis: the Multiscale Approach, and Astronomical Image and Data Analysis.

V. J. Martínez received his Ph.D. degree in mathematics from the University of Valencia in 1989, after preparing his thesis at NORDITA, Copenhagen, where his Adviser was Bernard Jones. In 1991, he got a permanent position at the University of Valencia as an Associate Professor in astronomy and astrophysics. He is currently the Director of the Observatori Astronómic dela Universitat de Valencia. He works on the statistical

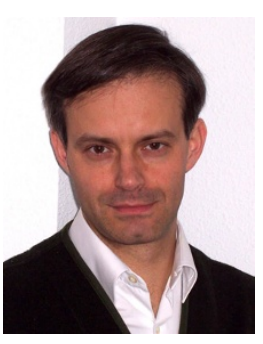
properties of the large-scale structure of the Universe. He is also involved in observational projects to study the mass and extent of dark halos around elliptical galaxies. He is the coauthor, with E. Saar, of the book Statistics of the Galaxy Distribution. 
D. L. Donoho is Anne T. and Robert M. Bass Professor in the humanities and sciences at Stanford University. He received his A.B. degree in statistics at Princeton University where his thesis Adviser was John W. Tukey and his Ph.D. degree in statistics at Harvard University, where his thesis adviser was Peter J. Huber. He is a Member of the US National Academy of Sciences and of the American Academy of Arts and Sciences.

O. Levi is a faculty member in the Department of Industrial Engineering and Management, Ben-Gurion University of the Negev, Beer Sheva, Israel. He received the B.S. degree in mathematics and industrial engineering and the M.S. degree in industrial engineering from Ben-Gurion University, Israel. He earned his Ph.D. degree in scientific computing and computational mathematics from Stanford University in 2004. His thesis title was entitled "Multiscale geometric analysis of 3-D data sets" and he worked under the supervision of Professor D. Donoho. His fields of interest include scientific computing, matrix computation, discrete Fourier analysis, and MSG algorithms.

P. Querre has an Engineering degree from the Institut National Polytechnique de Toulouse. He has been working for the last five years at the Service d'Astrophysique, Saclay, on image and signal processing applications. He got involved in the development of new methods of 2D and 3D redundant multiscale transforms. His main research interests are signal and image processing algorithms.

E. Saar received his Cand. Sci. (Ph.D.) degree in theoretical physics at Tartu University in 1971, and his Dr. Astr. degree at the same university in 1991. He has worked all his life at Tartu Observatory, at positions ranging from a Programmer to a Vice Director. Currently he is the Head of the Cosmology Department. He has written papers on general relativity (inhomogeneous cos-

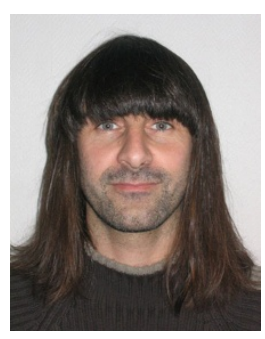
mologies), physics of galaxies (spiral struc-

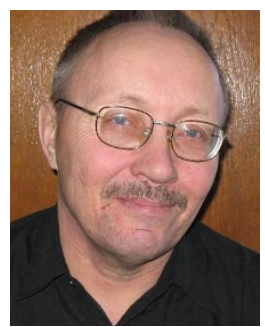
ture, gaseous halos), atmospheric physics (space experiments), and cosmology (dark matter, large-scale structure). His main interests at present are the statistics of the cosmological large-scale structure and of the CMB, and numerical modelling of the formation of this structure. He is a coauthor, with V. J. Martínez, of the book Statistics of the Galaxy Distribution. 